\title{
Local free-fall Temperature of modified Schwarzschild black hole in rainbow spacetime
}

\author{
Yong-Wan Kim¹ and Young-Jai Park $\oplus^{\Uparrow 2}$ \\ ${ }^{1}$ Research Institute of Physics and Chemistry, \\ Chonbuk National University, Jeonju 54896, Korea, \\ ${ }^{2}$ Department of Physics, Sogang University, Seoul 04107, Korea
}

September 19, 2021

\begin{abstract}
We obtain a $(5+1)$-dimensional global flat embedding of modified Schwarzschild black hole in rainbow gravity. We show that local freefall temperature in rainbow gravity, which depends on different energy $\omega$ of a test particle, is finite at the event horizon for a freely falling observer, while local temperature is divergent at the event horizon for a fiducial observer. Moreover, these temperatures in rainbow gravity satisfy similar relations to those of the Schwarzschild black hole except overall factor $g(\omega)$, which plays a key role of rainbow functions in this embedding approach.
\end{abstract}

PACS numbers: 04.70.Dy, 04.20.Jb, 04.62.+v

Keywords: Modified Schwarzschild spacetime, Rainbow gravity, Global flat embedding, Unruh effect

\footnotetext{
*Electronic address: ywkim65@gmail.com

${ }^{\dagger}$ Electronic address: yjpark@sogang.ac.kr
} 


\section{Introduction}

Hawking discovered that a black hole can be described by the characteristic temperature $T_{H}$ as seen by an asymptotic observer [1]. On the other hand, a fiducial observer staying at a finite distance from a black hole sees thermal radiation given by the Tolman temperature [2]. Furthermore, Unruh [3] showed that a uniformly accelerated observer with a proper acceleration in flat spacetime detects thermal radiation at the Unruh temperature. These two effects are, however, closely related each other, i.e., the Hawking effect for a fiducial observer in a curved spacetime can be considered as the Unruh effect for a uniformly accelerated observer in a higher dimensional flat spacetime. This provides us a unified derivation of temperature firstly given by Deser and Levin [4, and after that there have been much work on a variety of curved spacetimes [5, 6, 17, 8, 9, 10, 11, 12, 13, 14 in this line of the global embedding Minkowskian spacetime (GEMS). Later, this GEMS approach has been used to define a local temperature for a freely falling observer outside various black hole spacetimes [15, 16, 17].

On the other hand, there has also been much attention to modified dispersion relations (MDR) in gravity's rainbow, which can be viewed as an extension of doubly special relativity [18] in curved spacetime. In particular, Magueijo and Smolin [19, 20] proposed that the spacetime background felt by a test particle would depend on its energy $\omega$ such that the energy of the test particle deforms the background geometry and consequently the dispersion relation as follows

$$
\omega^{2} f^{2}\left(\omega / \omega_{p}\right)-p^{2} g^{2}\left(\omega / \omega_{p}\right)=m^{2},
$$

where $p, m, \omega_{p}$ are the momentum, the mass of the test particle, the Planck energy, respectively. Here, the rainbow functions of $f\left(\omega / \omega_{p}\right), g\left(\omega / \omega_{p}\right)$ with the conditions $\lim _{\omega \rightarrow 0} f\left(\omega / \omega_{p}\right)=1$ and $\lim _{\omega \rightarrow 0} g\left(\omega / \omega_{p}\right)=1$ at low energies are determined depending on the specific models. Since then great efforts have been devoted to the rainbow gravity related to the gravity and other stimulated work at the Planck scale [21, 22, 23, 24, 25, 26, 27, 28, 29, 30, 31, 32. In connection with the black hole thermodynamics, even though the spectrum emitted at infinite from a black hole would be only marginally affected by the MDR [33], there have been much work on black hole physics in the rainbow gravity [34, 35, 36, 37, 38, 39]. In fact, the temperature as well as the entropy of a black hole probed by a test particle receives energy dependent corrections due to the modification of the MDR. Recently, the black hole thermodynamics in 
the rainbow gravity was studied with the following choice of rainbow functions [40, 41, 42, 43] among many others [19, 28, 31, 35, 36, 37, 39],

$$
f\left(\omega / \omega_{p}\right)=1, \quad g\left(\omega / \omega_{p}\right)=\sqrt{1-\eta\left(\omega / \omega_{p}\right)^{n}}
$$

where $n$ is a positive integer and $\eta$ is a constant of order unity. This was originally motivated from a quantum spacetime phenomenology [44, and is compatible with some of the results in Loop Quantum Gravity [45] and $\kappa$ Minkowski noncommutative spacetime [46]. From now on, we will take $\omega_{p}=1$, for simplicity, unless mentioned otherwise.

In this paper, we wish to study the GEMS of the modified Schwarzschild black hole in the rainbow gravity. We show that the energy dependent local free-fall temperature is finite at the event horizon for a freely falling observer, while the energy dependent local temperature is divergent for a fiducial observer. In section 2, we briefly review the structure of the GEMS of the Schwarzschild spacetime and its local free-fall temperature. In section 3, we apply this GEMS approach to the modified Schwarzschild black hole in the rainbow gravity, and find their corresponding local free-fall temperature. As a result, we find that the temperatures in the modified Schwarzschild black hole keeps the same as those in the Schwarzschild black hole, except overall factor of $g(\omega)$. Our conclusions are drawn in section 4 .

\section{GEMS of the Schwarzschild black hole}

First, let us briefly recapitulate the GEMS embedding of the Schwarzschild spacetime with the metric

$$
\left(d s_{4}\right)^{2}=-h(r) d t^{2}+h^{-1}(r) d r^{2}+r^{2}\left(d \theta^{2}+\sin ^{2} \theta d \phi^{2}\right),
$$

which can be embedded into a $(5+1)$-dimensional Minkowskian spacetime of

$$
\left(d s_{6}\right)^{2}=\eta_{I J} d z^{I} d z^{J}
$$

with $h(r)=1-2 M / r$ and a metric $\eta_{I J}=\operatorname{diag}(-1,1,1,1,1,1)$. The two metrics are related through the embedding functions $z^{I}=z^{I}\left(x^{\mu}\right)$ with the metric $g_{\mu \nu}=\eta_{I J} \partial z^{I} / \partial x^{\mu} \partial z^{J} / \partial x^{\nu}$. Then, $z^{I}\left(x^{\mu}\right)$ [47] are explicitly given by

$$
z^{0}=k_{H}^{-1} \sqrt{h(r)} \sinh \left(k_{H} t\right)
$$




$$
\begin{aligned}
z^{1} & =k_{H}^{-1} \sqrt{h(r)} \cosh \left(k_{H} t\right) \\
z^{2} & =\int d r \sqrt{\frac{2 M\left(r^{2}+2 M r+4 M^{2}\right)}{r^{3}}} \\
z^{3} & =r \sin \theta \cos \phi \\
z^{4} & =r \sin \theta \sin \phi \\
z^{5} & =r \cos \theta
\end{aligned}
$$

Here, we denote the surface gravity $k_{H}$ on the event horizon of the Schwarzschild black hole as

$$
k_{H}=-\frac{1}{2} \lim _{r \rightarrow r_{H}} \sqrt{\frac{-g^{11}}{g^{00}}} \frac{\left(g^{00}\right)^{\prime}}{g^{00}}=\frac{1}{2 r_{H}},
$$

where the event horizon $r_{H}$ is given by $2 M$.

Then, a uniformly accelerating observer follows a hyperbolic trajectory in the $(5+1)$-dimensional flat spacetime described by a proper acceleration

$$
a_{6}^{-2}=\left(z^{1}\right)^{2}-\left(z^{0}\right)^{2}=16 M^{2} h(r) .
$$

Thus, the Unruh temperature [3] can be easily read as

$$
T_{U}=\frac{a_{6}}{2 \pi}=\frac{1}{8 \pi M \sqrt{h(r)}} .
$$

This corresponds to the local temperature measured by a fiducial observer staying at a finite distance from the black hole, the so-called fiducial temperature

$$
T_{\mathrm{FID}}=\frac{T_{H}}{\sqrt{-g_{00}}}
$$

where the Hawking temperature $T_{H}$ is measured by an asymptotic observer

$$
T_{H}=\frac{1}{8 \pi M} .
$$

As a result, one can say that the Hawking effect for a fiducial observer in the black hole spacetime is just the Unruh effect for a uniformly accelerated observer in a higher-dimensional flat spacetime.

Next, let us consider a freely falling observer who has been dropped from rest at $r=r_{0}$ and at $\tau=0$. For a freely falling observer, there are turning points [15, 16, 17] of radial geodesics where a freely falling observer is momentarily at rest with respect to black holes. 
For a Killing vector of $K^{\mu}=\left(\partial_{t}\right)^{\mu}=(1,0,0,0)$ related to the energy conservation, we have a constant of motion

$$
K_{\mu} \frac{d x^{\mu}}{d \lambda}=-h(r) \frac{d x^{\mu}}{d \lambda} \equiv-E
$$

Then, timelike equation

$$
1=-g_{\mu \nu} \frac{d x^{\mu}}{d \lambda} \frac{d x^{\nu}}{d \lambda}
$$

becomes

$$
1=h(r)\left(\frac{d t}{d \lambda}\right)^{2}-h^{-1}(r)\left(\frac{d r}{d \lambda}\right)^{2}
$$

along with an equatorial plane $\theta=\pi / 2$. From Eqs. (2.14) and (2.16), one can find the following orbit equations [15, 16, 17]

$$
\begin{aligned}
& \frac{d t}{d \tau}=\frac{\sqrt{h\left(r_{0}\right)}}{h(r)} \\
& \frac{d r}{d \tau}=-\sqrt{h\left(r_{0}\right)-h(r)} .
\end{aligned}
$$

Therefore, one can obtain the $\widetilde{a}_{6}$ acceleration at the turning point $r=r_{0}$ given by

$$
\left(\widetilde{a}_{6}\right)^{2}=\eta_{I J} \widetilde{a}^{I} \widetilde{a}^{J}=\frac{r^{3}+2 M r^{2}+4 M^{2} r+8 M^{3}}{16 M^{2} r^{3}} .
$$

Now, the local free-fall temperature measured by the freely falling observer at rest (FFAR) can be obtained as

$$
T_{\mathrm{FFAR}}=\frac{\widetilde{a}_{6}}{2 \pi}=\sqrt{\sum_{n=0}^{3}\left(\frac{2 M}{r}\right)^{n}} T_{H} .
$$

In Figure 1 we plot the two temperatures $T_{F I D}$ and $T_{F F A R}$ as functions of the radial variable $r$. Note that as shown in Figure 1, the local free-fall temperature at the event horizon is finite as $T_{\mathrm{FFAR}}=2 T_{H}$, while the local fiducial temperature $T_{\text {FID }}(2.12)$ for the fiducial observer diverges as $r \rightarrow r_{H}$ as shown by Brynjolfsson and Thorlacius. [15] 


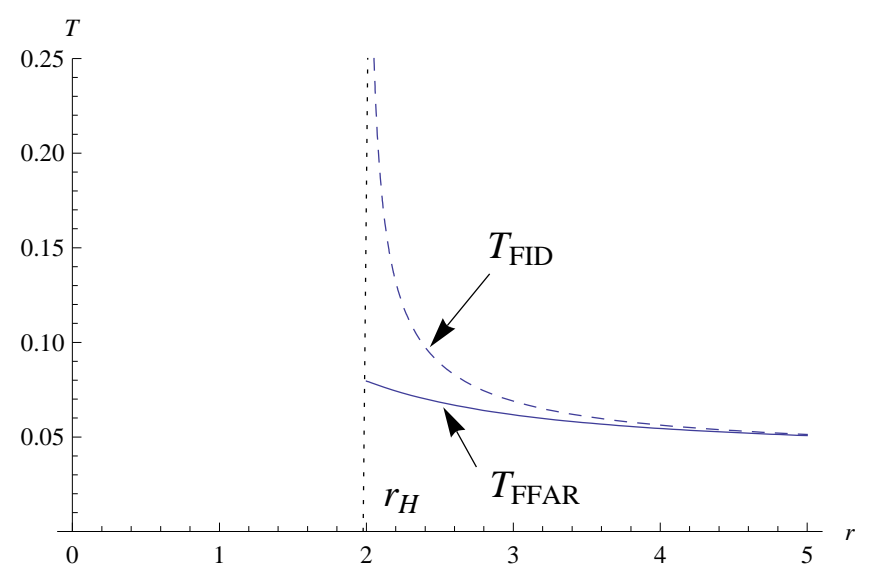

Figure 1: The local temperatures $T_{F F A R}$ and $T_{F I D}$ plotted as a function of the radial variable $r$. The free-fall temperature $T_{F F A R}$ seen by a freely falling observer remains finite at the horizon, while the fiducial temperature $T_{F I D}$ seen by an observer staying at a finite distance from the horizon blows up.

\section{Modified Schwarzschild black hole in rain- bow spacetime}

Now, we are ready to study the modified Schwarzschild black hole in the rainbow spacetime, which is described by the metric

$$
\left(d s_{4}^{\omega}\right)^{2}=-\frac{1}{f^{2}(\omega)} h(r) d t^{2}+\frac{1}{g^{2}(\omega)} h^{-1}(r) d r^{2}+\frac{r^{2}}{g^{2}(\omega)}\left(d \theta^{2}+\sin ^{2} \theta d \phi^{2}\right) .
$$

This spacetime can be embedded into a $(5+1)$-dimensional Minkowskian spacetime

$$
\left(d s_{6}^{\omega}\right)^{2}=\eta_{I J} d z^{\prime I} d z^{J}
$$

with a metric $\eta_{I J}=\operatorname{diag}(-1,1,1,1,1,1)$. Explicitly, the embedding functions $z^{\prime I}\left(x^{\mu}\right)$ are given by the following coordinate transformations depending on different energy $\omega$ of the probe

$$
\begin{aligned}
z^{\prime 0} & =\frac{\sqrt{h(r)}}{k_{H}^{\omega} f(\omega)} \sinh \left(k_{H}^{\omega} t\right), \\
z^{\prime 1} & =\frac{\sqrt{h(r)}}{k_{H}^{\omega} f(\omega)} \cosh \left(k_{H}^{\omega} t\right),
\end{aligned}
$$




$$
\begin{aligned}
z^{\prime 2} & =\frac{1}{g(\omega)} \int d r \sqrt{\frac{2 M\left(r^{2}+2 M r+4 M^{2}\right)}{r^{3}}}, \\
z^{\prime 3} & =\frac{1}{g(\omega)} r \sin \theta \cos \phi \\
z^{\prime 4} & =\frac{1}{g(\omega)} r \sin \theta \sin \phi \\
z^{\prime 5} & =\frac{1}{g(\omega)} r \cos \theta .
\end{aligned}
$$

Note here that the surface gravity $k_{H}^{\omega}$ on the event horizon in the rainbow gravity, is defined by

$$
k_{H}^{\omega}=\frac{g(\omega)}{f(\omega)} k_{H} .
$$

Then, the Hawking temperature $T_{H}^{\omega}$ measured by an asymptotic observer can be written

$$
T_{H}^{\omega}=\frac{k_{H}^{\omega}}{2 \pi}=\frac{g(\omega)}{f(\omega)} T_{H}
$$

Note that Eq. (3.10) indicates that the temperature of the modified Schwarzschild black hole is different for a probe with different energy $\omega$.

It seems appropriate to comment that the factors $k_{H}^{\omega} f(\omega)$ in Eqs. (3.3) and (3.4) can be rewritten as $g(\omega) k_{H}$. Then, we have the relation between the line elements $d s_{6}^{\omega}$ and $d s_{6}$ as

$$
\left(d s_{6}^{\omega}\right)^{2}=g^{-2}(\omega)\left(d s_{6}\right)^{2} .
$$

Thus, it seems particularly that only the $g(\omega)$ plays the key role of the two rainbow functions in the GEMS embedding.

On the other hand, an observer, who is uniformly accelerated in the $(5+1)$ dimensional flat spacetime, follows a hyperbolic trajectory described by a proper acceleration

$$
\left(a_{6}^{\omega}\right)^{-2}=\left(z^{1}\right)^{2}-\left(z^{\prime 0}\right)^{2}=g^{-2}(\omega)\left(a_{6}\right)^{-2} .
$$

Then, the Unruh temperature can be read as

$$
T_{U}^{\omega}=\frac{a_{6}^{\omega}}{2 \pi}=g(\omega) T_{U}
$$


In fact, this corresponds to the fiducial temperature measured by a fiducial observer at a finite distance from the black hole

$$
T_{\mathrm{FID}}^{\omega}=\frac{T_{H}^{\omega}}{\sqrt{-g_{00}^{\omega}}}=g(\omega) T_{\mathrm{FID}}
$$

As a result, the Hawking effect for a fiducial observer in the rainbow spacetime can also be said to be the Unruh effect for a uniformly accelerated observer in a higher-dimensional flat spacetime as like in the Schwarzschild spacetime. Moreover, both of them are only proportional to the rainbow function $g(\omega)$ in common.

Now, consider a freely falling observer who is dropped from rest at $r=r_{0}$ and $\tau=0$. Making use of a constant of motion as in Eq. (2.14) for the modified Schwarzschild spacetime in the rainbow gravity

$$
\begin{aligned}
& \frac{d t}{d \tau}=\frac{f(\omega) \sqrt{h\left(r_{0}\right)}}{h(r)} \\
& \frac{d r}{d \tau}=-g(\omega) \sqrt{h\left(r_{0}\right)-h(r)}
\end{aligned}
$$

one can find the acceleration $\widetilde{a}_{6}^{\omega}$ for the freely falling observer

$$
\left(\widetilde{a}_{6}^{\omega}\right)^{2}=g^{2}(\omega)\left(\widetilde{a}_{6}\right)^{2}
$$

where $\widetilde{a}_{6}$ is the acceleration given by (2.19). Therefore, the local free-fall temperature seen by a freely falling observer at rest can be written by

$$
T_{\mathrm{FFAR}}^{\omega}=\frac{\widetilde{a}_{6}^{\omega}}{2 \pi}=g(\omega) \sqrt{\sum_{n=0}^{3}\left(\frac{2 M}{r}\right)^{n}} T_{H}=g(\omega) T_{\mathrm{FFAR}} .
$$

In Figure 2, we plot the two temperatures $T_{F F A R}^{\omega}$ and $T_{F I D}^{\omega}$ as functions of the radial variable $r$ and a fixed $\omega=0.8$. It is important to note that similar to the Schwarzschild case, the local free-fall temperature at the event horizon is finite as $T_{\text {FFAR }}^{\omega}=2 g(\omega) T_{H}$, while the local temperature $T_{\text {FID }}^{\omega}$ for the fiducial observer diverges as $r \rightarrow r_{H}$. In Figure 3 and 4 , we also plot the local temperatures of $T_{F F A R}^{\omega}$ and $T_{F I D}^{\omega}$, respectively, as functions of the radial variable $r$ and $\omega$ with $\eta=1, n=2$, which show that the local free-fall temperature $T_{F F A R}^{\omega}$ is less sensitive to the energy $\omega$ of the probe. 


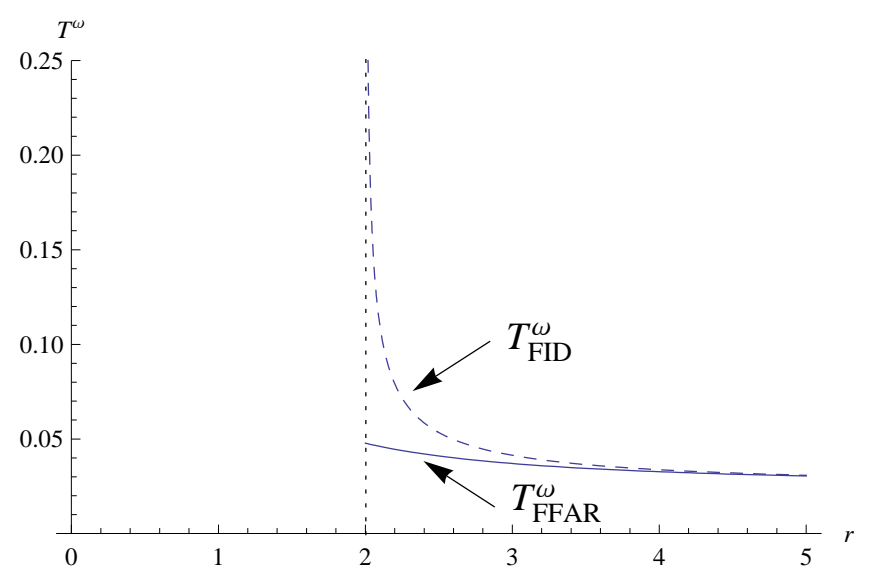

Figure 2: The local temperatures $T_{F F A R}^{\omega}$ and $T_{F I D}^{\omega}$ plotted as functions of the radial variable $r$ with $\eta=1, \omega=0.8$, and $n=2$. Similar to the Schwarzschild case, the free-fall temperature $T_{F F A R}^{\omega}$ also remains finite at the horizon while the fiducial temperature $T_{F I D}^{\omega}$ blows up.

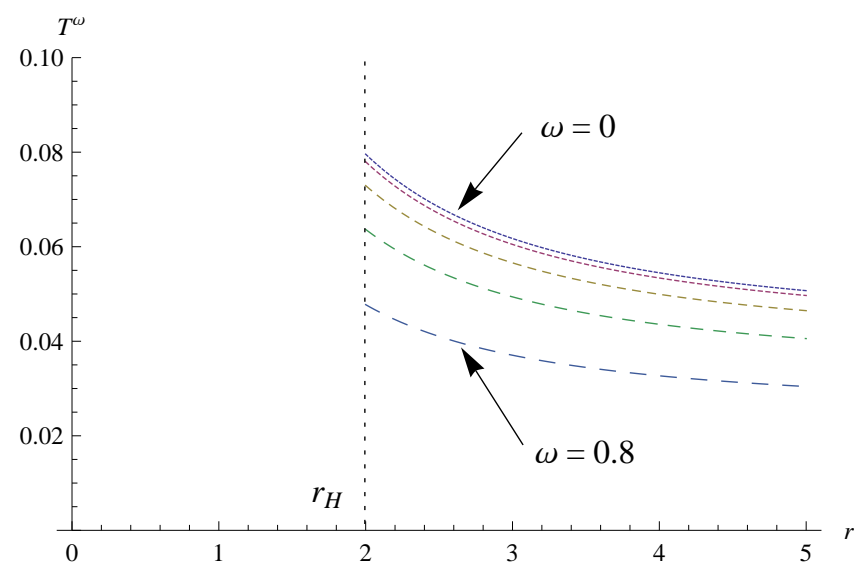

Figure 3: The local free-fall temperature $T_{F F A R}^{\omega}$ plotted as functions of the radial variable $r$ with $\eta=1, n=2$, and $\omega=0,0.2,0.4,0.6,0.8$, respectively. The curve with $\omega=0$ corresponds to $T_{F F A R}$ in Eq. (2.20). The curves show that the local free-fall temperatures become finite as $r \rightarrow r_{H}$ regardless of the energy $\omega$ of a test particle. 


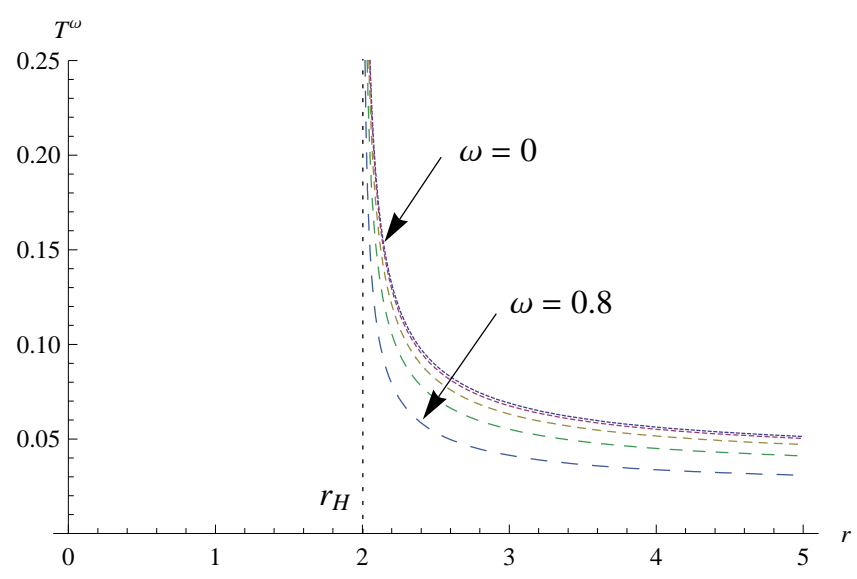

Figure 4: The local fiducial temperature $T_{F I D}^{\omega}$ plotted as functions of the radial variable $r$ with $\eta=1, n=2$, and $\omega=0,0.2,0.4,0.6,0.8$, respectively. The curve with $\omega=0$ corresponds to $T_{F I D}$ in Eq. (2.12). They show that the local fiducial temperatures diverge as $r \rightarrow r_{H}$ regardless of the energy $\omega$ of a test particle.

\section{Conclusions}

In this paper, we have obtained a $(5+1)$-dimensional global flat embedding of the modified Schwarzschild black hole in rainbow gravity. We have shown that with the proper choice of the rainbow functions (1.2), the local free-fall temperature for a freely falling observer is finite at the event horizon, while the local temperature for a fiducial observer is divergent, which are similar to the case of the Schwarzschild black hole, except that these temperatures also depend on different energy $\omega$ of a test particle.

On the other hand, it seems to be important to note that in the GEMS approach all the local temperatures of $T_{U}^{\omega}, T_{F I D}^{\omega}$, and $T_{F F A R}^{\omega}$ depend only on the $g(\omega)$ of the two rainbow functions in Eq. (1.2).

\section{References}

[1] S. W. Hawking, Commun. Math. Phys. 43, 199 (1975) [Commun. Math. Phys. 46, 206 (1976)]. 
[2] R. C. Tolman, "Relativity, Thermodynamics and Cosmology", New York, Dover Publication (1987).

[3] W. G. Unruh, Phys. Rev. D 14, 870 (1976).

[4] S. Deser and O. Levin, Phys. Rev. D 59, 064004 (1999).

[5] Y. -W. Kim, Y. -J. Park and K. -S. Soh, Phys. Rev. D 62, 104020 (2000).

[6] S. -T. Hong, Gen. Rel. Grav. 36, 1919 (2004).

[7] H. -Z. Chen, Y. Tian, Y. -H. Gao and X. -C. Song, JHEP 0410, 011 (2004).

[8] N. L. Santos, O. J. C. Dias and J. P. S. Lemos, Phys. Rev. D 70, 124033 (2004).

[9] R. Banerjee and B. R. Majhi, Phys. Lett. B 690, 83 (2010).

[10] R. G. Cai and Y. S. Myung, Phys. Rev. D 83, 107502 (2011).

[11] B. R. Majhi, arXiv:1110.6008 [gr-qc].

[12] B. Hu and H. F. Li, Mod. Phys. Lett. A 27, 1250002 (2012).

[13] S. A. Paston, JHEP 1406, 122 (2014).

[14] S. A. Paston, Class. Quant. Grav. 32, 145009 (2015).

[15] E. J. Brynjolfsson and L. Thorlacius, JHEP 0809, 066 (2008).

[16] Y. W. Kim, J. Choi and Y. J. Park, Int. J. Mod. Phys. A 25, 3107 (2010).

[17] Y. W. Kim, J. Choi and Y. J. Park, Phys. Rev. D 89, 044004 (2014).

[18] G. Amelino-Camelia, Int. J. Mod. Phys. D 11, 35 (2002).

[19] J. Magueijo and L. Smolin, Phys. Rev. D 67, 044017 (2003).

[20] J. Magueijo and L. Smolin, Class. Quant. Grav. 21, 1725 (2004).

[21] S. Liberati, S. Sonego and M. Visser, Phys. Rev. D 71, 045001 (2005).

[22] P. Galan and G. A. Mena Marugan, Phys. Rev. D 72, 044019 (2005). 
[23] J. Hackett, Class. Quant. Grav. 23, 3833 (2006).

[24] Y. Ling, JCAP 0708, 017 (2007).

[25] Y. Ling, S. He and H. -b. Zhang, Mod. Phys. Lett. A 22, 2931 (2007).

[26] F. Girelli, S. Liberati and L. Sindoni, Phys. Rev. D 75, 064015 (2007).

[27] Y. Ling and Q. Wu, Phys. Lett. B 687, 103 (2010).

[28] R. Garattini and G. Mandanici, Phys. Rev. D 85, 023507 (2012).

[29] R. Garattini and F. S. N. Lobo, Phys. Rev. D 85, 024043 (2012).

[30] G. Amelino-Camelia, M. Arzano, G. Gubitosi and J. Magueijo, Phys. Rev. D 88, 041303 (2013).

[31] A. Awad, A. F. Ali and B. Majumder, JCAP 1310, 052 (2013).

[32] J. D. Barrow and J. Magueijo, Phys. Rev. D 88, 103525 (2013).

[33] W. G. Unruh, Phys. Rev. Lett. 46, 1351 (1981).

[34] G. Amelino-Camelia, M. Arzano, Y. Ling and G. Mandanici, Class. Quant. Grav. 23, 2585 (2006).

[35] Y. Ling, X. Li and H. b. Zhang, Mod. Phys. Lett. A 22, 2749 (2007).

[36] C. Z. Liu and J. Y. Zhu, Gen. Rel. Grav. 40, 1899 (2008).

[37] J. J. Peng and S. Q. Wu, Gen. Rel. Grav. 40, 2619 (2008).

[38] H. Li, Y. Ling and X. Han, Class. Quant. Grav. 26, 065004 (2009).

[39] R. Garattini, Phys. Lett. B 685, 329 (2010).

[40] A. F. Ali, Phys. Rev. D 89, 104040 (2014).

[41] Y. Gim and W. Kim, JCAP 1410, 003 (2014).

[42] A. F. Ali, M. Faizal and M. M. Khalil, Phys. Lett. B 743, 295 (2015).

[43] Y. Gim and W. Kim, JCAP 1505, 002 (2015). 
[44] G. Amelino-Camelia, Living Rev. Rel. 16, 5 (2013).

[45] R. Gambini and J. Pullin, Phys. Rev. D 59, 124021 (1999).

[46] J. Lukierski, H. Ruegg and W. J. Zakrzewski, Annals Phys. 243, 90 (1995).

[47] C. Fronsdal, Phys. Rev. 116, 778 (1959). 